\title{
SU-F-T-108: Texture Analysis for Toxicity Prediction From Dose Surface Maps.
}

Witztum $\mathrm{A}^{1}$, Warren $\mathrm{S}^{1}$, Partridge $\mathrm{M}^{1}$, Hawkins $\mathrm{M}^{1}$.

Author information

${ }^{1}$ CRUK/MRC Oxford Institute for Radiation Oncology, Department of Oncology, University of Oxford, Oxford, UK.

\section{Abstract \\ PURPOSE:}

To find spatial features that best predict acute duodenal related toxicity grade $\geq 3$ from $2 \mathrm{D}$ dose surface maps in pancreatic chemoradiotherapy.

\section{METHODS:}

Nineteen patients from the ARCII trial (EudraCT 2008-006302-42) treating locally-advanced pancreatic cancer (LAPC) with chemoradiotherapy were analysed. Previous work considering traditional dose-volume and dose-surface metrics as predictors had little power. We investigate the use of texture analysis to improve predictive capabilities. 2D duodenal dose surface maps (DSMs) were converted to 4, 8, and 16 gray (dose) levels. A gray-level co-occurrence matrix (GLCM) was produced and statistics (contrast, correlation, energy and homogeneity) were extracted. ROC curves were produced for each statistic for three different gray level maps $(4,8$, and 16$)$. The area under curve (AUC) was recorded and used to find the best predictor for acute duodenal related toxicity grade $\geq 3$.

\section{RESULTS:}

For all GLCM statistics there were only small differences in AUC between 4, 8 and 16 gray levels. Homogeneity had an AUC of $0.87,0.88$ and 0.85 for 4,8 and 16 gray levels respectively. Using cut point analysis on homogeneity using 8 gray levels it was found that a value of 0.945 correctly predicted toxicity for $17 / 19$ patients (4/6 patients with, $13 / 13$ patients without toxicity). The two incorrectly predicted patients had a mean dose of $59 \mathrm{~Gy}$ and $28 \mathrm{~Gy}$. The AUC for traditional dosevolume ( $\mathrm{V} 45=0.57, \mathrm{~V} 50=0.60, \mathrm{~V} 55=0.47$ ) and dose-surface metrics ( 455 (surface area receiving at least $45 \mathrm{~Gy}$ ) $=0.50, \mathrm{~S} 50=0.57, \mathrm{~S} 55=0.49$ ) were much lower.

\section{CONCLUSION:}

Use of texture analysis improves our ability to predict grade $\geq 3 \mathrm{Gl}$ toxicity in pancreatic chemoradiotherapy. The additional spatial information may give better predictors than traditional dose-volume and dose-surface metrics. The mechanism behind this predicted toxicity will be studied on a larger patient set. Alon Witztum is supported by an MRC/Gray Institute DPhil Studentship. Sam Warren and Mike Partridge are supported by CRUK grant C5255/A15935. Maria Hawkins received an MRC Fellowship MC_PC_12001/2. 